\title{
Enhanced oxygen evolution reaction by stacking single-crystalline freestanding $\mathrm{SrRuO3}$
}

\section{Qixiang Wang}

Huan Liu

Harbin Institute of Technology, Jilin Normal University

Bin He ( $\nabla$ sdy_heb@ujn.edu.cn )

University of Jinan https://orcid.org/0000-0003-1561-2467

Ji Qi

Harbin Institute of Technology, Jilin Normal University

Di Wang

University of Jinan

Hang Xu

Harbin Institute of Technology, Jilin Normal University

Nana Zhang

University of Jinan

Jie Wang

University of Jinan, Harbin Institute of Technology

Yanan Chen

University of Jinan

Zhihong Wang

Harbin Institute of Technology

Zhengjia Wang

Harbin Institute of Technology

Limei Zheng

Shandong University

Ming Feng

Jilin Normal University

Weiming Lü

Harbin Institute of Technology

Shishen Yan

Shandong University https://orcid.org/0000-0002-7327-9968

Article 
Keywords:

Posted Date: September 11th, 2021

DOI: https://doi.org/10.21203/rs.3.rs-847470/v1

License: (c) (1) This work is licensed under a Creative Commons Attribution 4.0 International License. Read Full License 


\section{Abstract}

Transition metal oxide thin film has recently become an attractive platform for enhanced catalytic activity and to perceive the fundamental functions of $d$ electron structure and charge transfer processes. Owing to the long-range lattice ordering and accurate stoichiometry, the single-crystalline transition metal oxide thin film enables the mechanism discussion of electrochemistry down to the atomic level. However, it is technically unviable in the fabrication of transition metal oxide thin film with a substantial surface area and strain condition. Here we report the oxygen evolution reaction enhancement by a stack of multilayer $\mathrm{SrRuO}_{3}$ featured with single-crystallinity, flexibility, and stackability, which was achieved from the rigid heterostructure via a water-dissolution of $\mathrm{Sr}_{3} \mathrm{Al}_{2} \mathrm{O}_{6}$ sacrifice interlayers. The controllable stack of multilayer $\mathrm{SrRuO}_{3}$ and the emergent high-spin state $t_{2 g}(3 \uparrow) e_{g}(1 \uparrow)$ of Ru efficiently enhances the oxygen evolution reaction activity. Our study provides an approach for fine manipulation of single-crystalline freestanding transition metal oxide morphologically, and an efficient strategy aiming at the extreme enhancement of the electrochemically active surface area and strain condition.

\section{Introduction}

Functional perovskite transition metal oxides (TMO) have emerged as a promising candidate in various water splitting processes as its designable $d$ electron configuration and charge transfer energy between the transition metal and oxygen or hydroxyl, benefiting to a low cost, considerable activity, and stable catalyst $^{1-3}$. Among several decent material forms in oxygen evolution reaction (OER) investigations, single-crystalline TMO epitaxial thin film and heterostructure has been established as one important path to gain the fundamental links, even at the atomic level, between $d$ orbitals in the varied lattice structure and active site without a serious confusion of determining the specific surface area ${ }^{4-9}$. The interfacial lattice structure of rigid TMO heterostructure greatly influences the $d$ electron configuration by manipulating the $\mathrm{M}-\mathrm{O}$ octahedra. As a catalyst, it is excepted that a corresponding OER variation will be presented owing to a change of the ability of charge transfer such as in $\mathrm{LaCoO}_{3}, \mathrm{NdNiO}_{3}$, and $\mathrm{LaNiO}_{3}$, etc $^{10-12}$. In the conventional frame of improving catalytic activity, the enlargement of the electrochemical active surface area (ECSA) of a catalyst, such as in alloy or self-supporting electrocatalyst ${ }^{13,14}$, is widely explored by nanoparticle and porosity processes ${ }^{15,16}$. However, these strategies are inapplicable for the epitaxial single-crystalline TMOs as their lattice ordering and stoichiometry will be destructed and obscured. Besides the strain by interfacial lattice mismatch in rigid TMO thin films is limited in epitaxy, and the impact of interfacial strain may be extraordinarily puzzled due to it always relaxes in a scale of several unit cells, such as in $\mathrm{SrRuO}_{3}$ and $\mathrm{La}_{2 / 3} \mathrm{Sr}_{1 / 3} \mathrm{MnO}_{3}$, etc ${ }^{17,18}$. Thus up to now, a prominent enlargement of the single-crystalline TMO surface with conspicuous strain conditions is highly desired for OER investigations in the aspect of fundamentals and applications.

As a breakthrough, the nanometer-thick freestanding membrane of single-crystalline $\mathrm{La}_{0.67} \mathrm{Sr}_{0.33} \mathrm{MnO}_{3}$ by buffered water-dissolvable $\mathrm{Sr}_{3} \mathrm{Al}_{2} \mathrm{O}_{6}$ (SAO) method was firstly implemented by Lu et a ${ }^{19}$, then excellent mechanical properties were found in $\mathrm{BaTiO}_{3}(\mathrm{BTO})$ and $\mathrm{BiFeO}_{3}$ by Dong and $\mathrm{Ji}$ et al, where they can 
suffer up to $\sim 10.1 \%$ curvature change without a noticeable break of the perovskite ${ }^{20-22}$. Furthermore, the curling or related zigzag waveforms of freestanding $\mathrm{BaTiO}_{3}$ membranes were also demonstrated by Dong et al after a transfer to the Polydimethylsiloxane surface ${ }^{23}$. Thus this freestanding treatment of a single-crystalline TMO thin film enables us to obtain a flexible membrane that can be stacked or squeezed by a well-designed transfer and most importantly shed light on the possible surface area enlargement for a substantial enhancement of electrochemical catalysis. In addition, freestanding TMO also allows us to artificially manipulate the strain condition ${ }^{24-26}$. $\mathrm{SrRuO}_{3}(\mathrm{SRO})$ is an OER active transition metal oxide, and the electronic structure of SRO is sensitive to the strain condition which can trigger a high spin state for lowering the adsorption energy between SRO and oxygen intermediates ${ }^{27-31}$. As we have known that bulk SRO possesses a low-spin state of $\mathrm{Ru}^{4+}$ with an electron configuration of $t_{2 g}(3 \uparrow, 1 \downarrow)$ displaying a saturated magnetic moment of $2 \mu_{\mathrm{B}}$ per Ru theoretically ${ }^{32}$. A high-spin state of $t_{2 g}(3 \uparrow) e_{g}(1 \uparrow)$ occurs once the Ru-O octahedra is strained, the magnetic moment can surpass $3 \mu_{\mathrm{B}}$ per Ru according to previous experimental studies ${ }^{33,34}$. Although the Ru does not undergo a chemical valence change, introducing the occupancy of high-energy $e_{g}$ orbital could be an effective method to boost the OER performance ${ }^{35-37}$. Here in this study, aiming at a combination of the ECSA enlargement and strain engineering for an enhanced OER activity, we have performed a comprehensive investigation of the single-crystalline multi-layers of freestanding SROs on SRO, which can be achieved by water dissolving their supporting SAO interlayers. To maximumly strain the freestanding SRO, a cylindrical sharp was intentionally schemed out.

\section{Results And Discussions}

The fabrication of the single-layer $(n=1)$ cylindrical SRO (C-SRO) is schematically shown in Fig. 1a. An SRO layer was firstly deposited on (001)-oriented STO substrate as a conductive connecting layer (see Supplementary Fig. S1), followed by an SAO as the sacrifice layer. Then SRO/BTO/SRO was grown on SAO, here upper and lower SRO layers are asymmetric in thickness, 16 and $2 \mathrm{~nm}$ respectively, for producing an artificial strain distribution. As the water-dissolvable nature of SAO, SRO/BTO/SRO heterostructure and the rigid SRO will be separated once the sample is submerged into the deionized water. In the dissolution, the freestanding SRO/BTO/SRO gradually crackles and coils into the cylindrical sharp C-SRO due to the strain, as shown in Fig. 1b and c. After a complete solution of SAO, the C-SROs adhere to the rigid SRO and connect eventually with an aid of the exposed surface of rigid SRO.

We performed the X-ray diffraction measurements on rigid SRO/STO, and the SRO/BTO/SRO/SAO/SRO/STO heterostructure as shown in Fig. 1d. The rigid SRO layer is strictly epitaxial along with the (001) orientation. After inserting the SAO layer, the orientation of the epitaxial SRO/BTO/SRO/SAO/SRO/STO remains without any noticeable peaks of impurity structural phases. In Fig. 1e, the rigid SRO on STO exhibits a ferromagnetic to paramagnetic transition $T_{\mathrm{C}}$ at $\sim 170 \mathrm{~K}$ by the temperature-dependent magnetic measurement, also across $T_{\mathrm{c}}$, the transport, resistance versus 
temperature, of SRO shows a Fermi-liquid behavior to bad-metal transition (Fig. S1c). These physical properties of our SRO are consistent with previous reports 38,39 .

In Fig. 2a, we have shown the optical images of C-SRO layers (from $n=1,2,3,5$ and 10) on rigid SRO, and it is clear that the density of C-SRO increases with $n$, exhibiting a 3D stack SRO. After the waterdissolution, these samples were annealed at $400^{\circ} \mathrm{C}$ for 1 hour to make the SRO connection a strong adhesion instead of a weak one (such as Van der Waals force) for the stability in OER measurements as shown in Fig. S2. In Fig. 2b, the diameter ( $d$ ) of the C-SRO as a function of $n$ is presented, the $d$ slightly enlarges from $\sim 16.8$ to $\sim 18.6 \mu \mathrm{m}$ while $n=2$ to 10 . Before that the first C-SRO layer has a small $d \sim 13.7$ $\mu \mathrm{m}$. This change may come from the difference of supporting layers for C-SROs with $n=1$ and $n>1$. In principle, one may get any number of C-SRO layers by stacking SRO/BTO/SRO/SAO heterostructures in the epitaxy process. Before the OER activity tests, we examined the electrochemical double-layer capacitances $\left(C_{\mathrm{dl}}\right)$ by cyclic voltammetry $(\mathrm{CV})$ measurements in the Non-Faradaic current range at different scan rates (experimental details can be found in Fig. S3) to realize the C-SRO coverage effort from the view of ECSA. In Fig. 2c, we have found that $C_{\mathrm{dl}}$ as a function of $n$ exhibits a linear dependence benefiting from the stability of C-SRO, not only the shape, and diameter but also the connection. In addition, this result shows that our stack of C-SRO is an effective method to increase the surface area of single-crystalline TMO.

Utilizing a standard three-electrode configuration, the OER catalytic activities of our samples were evaluated in $1 \mathrm{~mol} / \mathrm{L} \mathrm{KOH}$ solution at room temperature. In Fig. 3a, linear sweep voltammetry (LSV) measurements of rigid SRO and with C-SRO (from $n=1$ to 10) coverage are shown. The current density (j) of $0.35 \mathrm{~mA} / \mathrm{cm}^{2}$ at $2 \mathrm{~V}$ of rigid SRO is found. By stacking C-SRO on it, the $j$ increases with $n$ monotonously from $n=1$ to 10 at a certain voltage. Further in Fig. 3b, the Tafel slopes, potential versus $\log (j)$ are plotted, where the Tafel slope reduces from 102 to $30 \mathrm{mV} / \mathrm{dec}$ with $n(0$ to 10$)$, indicating better reaction kinetics in the progress via C-SRO. Then the electrochemical impedance spectroscopy (EIS) Nyquist were measured at a frequency from $10^{5}$ to $0.01 \mathrm{~Hz}$ by $5 \mathrm{mV}$ amplitude AC signal as shown in Fig. 3c, as can be seen, the charge transfer resistance between a working electrode and electrolyte decreases with $n$, confirming that the coverage of C-SROs provides an enhanced charge transfer kinetics. In Fig. 3d-f, we have summarized the onsetpotentials, overpotentials, and Tafel slopes as a function of CSRO coverage to present an overall improvement of OER performance. The onsetpotential of rigid SRO is $\sim 1.900 \mathrm{~V}$, which is slightly larger than the previous reports of SRO thin films ${ }^{40,41}$. This is likely arisen by a faint difference of stoichiometry, it drops gradually with $n$ and reaches the minimum of $1.233 \mathrm{~V}$. Meanwhile the overpotential exhibits a similar regulation, by three-layer of C-SRO coverage, the overpotential can be $\sim 93 \%$ reduced from 0.92 to $0.06 \mathrm{~V}$ at $j=5 \mathrm{~mA} / \mathrm{cm}^{2}$. Throughout these overall performances, perhaps most interestingly, there is no saturation trend is seen up to $n=10$. It is not far to seek that these OER activity enhancements are originated from the enlargement of active surface area in part. In Fig. 3g, the electric field dependences of CV curves of rigid SRO and C-SRO $n=3$ and 10 with different scan rates are shown, detailed CV curves can be found in Fig.S3. The $j$ increases with scan rate and reaches $204,112,54$, and $27 \mu \mathrm{A} / \mathrm{cm}^{2}$ under $80,40,20$, and $10 \mathrm{mV} / \mathrm{s}$ in C-SRO $(n=10)$, which is 
roughly 18.5 times bigger than the one of rigid SRO. These results show that the C-SRO on rigid SRO is indeed a feasible strategy of the ECSA increase for an enhanced OER activity.

The forming of the cylindrical shape SRO comes from the BTO interlayer via the support effect, which enables us to achieve the stack of single-crystalline SROs. First of all, BTO is ferroelectric with a polarization $\sim 30 \mu \mathrm{C} / \mathrm{cm}^{2} 42$, the carrier of SRO will be depleted or accumulated at the BTO/SRO interface. According to the dielectric constant, and the carrier density of SRO, the screening length of SRO is $\sim 1.6 \AA$ which is smaller than the half unit cell ${ }^{43,44}$. So in such a scenario, the bound charge effect can neglect. Secondly, the BTO interlayer provides a lattice mismatch between two thickness asymmetric SROs for curling. Then the non-flat SRO may also contribute to the enhanced OER by extra electronic reconstruction, such as the appearance of a high-spin state, due to the strain effect. To clarify this, we compared the overpotential at $1 \mathrm{~mA}$ as a function of the SRO surface area in Fig. 4a. Here the surface area of C-SRO is evaluated by the value in the rigid heterostructure, say, before the water-dissolution. The overpotential decreases with the surface area in both rigid and C-SRO covered SRO, however, in the case of C-SRO, the overpotential is lower than epitaxial rigid SRO at a certain surface area, suggesting that this phenomenon is given by the strain effect, this difference is marked by grey area. In Fig. 4b, schematically the outer and inner SROs in C-SRO endure tensile and compressive strain respectively, the bending strain $\varepsilon$ in the bending direction is given by $\varepsilon=t / R$, where $t$ and $R$ are half of the thickness of SRO/BTO/SRO and the corresponding radius of curvature ${ }^{33}$. Roughly the outer and inner SROs bear $\pm 0.72 \%$ strain, in addition to an altering of the $d_{x y}, d_{x z}$ and $d_{y z}$ occupancy by the modification of $\mathrm{RuO}_{6}$ octahedra, the possible emerging high spin-state would contribute to a benefit of an enhanced OER activity. In Fig. 4c, we examine the magnetic field dependent magnetization of rigid SRO and C-SRO ( $n=3$ and 10). Based on the ideal stoichiometry, theoretical saturated magnetization $\left(M_{\mathrm{S}}\right)$ of $\mathrm{Ru}$ is two with the electron configuration of $t_{2 g}(3 \uparrow, 1 \downarrow)$, usually, the $M_{\mathrm{S}}$ of bulk SRO is always from $\sim 1.1$ to $\sim 1.6 \mu_{\mathrm{B}}$ per Ru owing to the electron delocalization associated with itinerancy $27,45,46$. In our rigid SRO, the $M_{\mathrm{S}}$ equals $\sim 1.61 \mu_{\mathrm{B}}$ per Ru under a parallel magnetic field, however, in contrast, the $M_{S}$ of C-SRO ( $n=3$ and 10$)$ exhibits a value of $\sim 2.24$ and $2.42 \mu_{\mathrm{B}}$ per Ru which indicates that the high-spin state occurs in our C-SRO. Then the occupancy of extra $e_{g}$ orbital provides an additional charge transfer path which could boost a further enhancement of OER activity. This conclusion is also testified by the X-ray photoelectron spectroscopy (XPS) measurement on the C-SRO and rigid SRO as shown in Fig. 4d. The binding energy of Ru $3 d_{3 / 2}$ shifts from $\sim 285.9 \mathrm{eV}$ of rigid SRO to $\sim 285.4 \mathrm{eV}$ of C-SRO. This indicates that the adsorption energy between C-SRO and oxygen intermediates can be lowered benefiting the OER performance ${ }^{40}$. Meanwhile, it implies that the electron occupancy of C-SRO is partially driven to a high-spin state 33,47 .

By achieving the enlarged ECSA and the high-spin state in stacked single-crystalline C-SRO, the enhanced OER performance is found, the overpotential of C-SRO $(n=10)$ is $\sim 60 \mathrm{mV}$ at $j=5 \mathrm{~mA} / \mathrm{cm}^{2}$, which is one order of magnitude smaller than the one of promising OER candidate TMO thin films, such as $\mathrm{LaNiO}_{3}{ }^{12}$, $\mathrm{LaCoO}_{3}{ }^{10}, \mathrm{Ba}_{0.5} \mathrm{Sr}_{0.5} \mathrm{Co}_{0.8} \mathrm{Fe}_{0.2} \mathrm{O}_{3-\delta}{ }^{48}$, and $\mathrm{IrO}_{2}{ }^{49}$. Besides, the Tafel slope of our C-SRO $(n=10)$ can be triggered to $\sim 30 \mathrm{mV} / \mathrm{dec}$, which is almost reduced by half comparing to theirs. 


\section{Conclusion}

In summary, aiming at the dilemma of the limitation of effective chemical reaction area of TMO epitaxial thin film, the stacking of freestanding SRO layers has been investigated to achieve a dramatical enhancement of OER activities. Meanwhile, the strain is introduced by coiling SRO to a cylinder sharp for a high-spin state $t_{2 g}(3 \uparrow) e_{g}(1 \uparrow)$ of SRO, which enriches the charge transfer process. Utilizing the sacrifice SAO interlayers and strain of SRO/BTO/SRO in rigid epitaxial heterostructures, after water-solution, freestanding C-SROs with electron configuration $t_{2 g}(3 \uparrow) e_{g}(1 \uparrow)$ in part on rigid SRO is obtained exhibiting an enlargement of SRO exposed surface. This greatly promotes OER activities in the aspect of onset potential, overpotential, and Tafel slope. Our study on the stack of freestanding SRO could initiate a promising method to scale up the active surface area of single-crystalline TMO without damage of lattice ordering and stoichiometry. Most importantly strain conditions can be well-engineered with the goal of a particular electronic structure.

\section{Declarations}

Acknowledgment: W. M. L. acknowledges financial support from the National Natural Science Foundation of China (No. 12074149). B. H. acknowledges support from the Shandong Provincial Natural Science Foundation (No. ZR2020QA057). M. F. acknowledges support from the National Natural Science Foundation of China (No. 51772126), Jilin Province Science and Technology Department Program (Nos. 20200201277JC, 20200201279JC, and 20190101009JH). L. M. Z. acknowledges support from the National Natural Science Foundation of China (No.52072218). S. S. Y. acknowledges support from the National Nature Science Foundation of China Grant No. 51871112, the Major Basic Research Projects of Shandong Province ZR2020ZD28, and the 111 Project B13029.

Author Contributions: B. H., W. L., L. Z. and S. Y. planned and supervised the research. Q. W. and H. L. performed sample fabrication and measurements. J. Q., D. W., H. X., N. Z., J. W. and Y. C. carried out the study and collected important background information. Z. W., Z. W. and M. F. provided assistance for data acquisition, data analysis and statistical analysis. B. H. and W. L. wrote the manuscript with help from all other authors.

\section{References}

1. Li, X. et al. Exceptional oxygen evolution reactivities on $\mathrm{CaCoO}_{3}$ and $\mathrm{SrCoO}_{3}$. Sci. Adv. 5, eaav6262 (2019).

2. Liu, J. S. et al. Tuning the electronic structure of $\mathrm{LaNiO}_{3}$ through alloying with strontium to enhance oxygen evolution activity. Adv. Sci. 6, 1901073 (2019).

3. Benson, E. E. et al. Dynamic tuning of a thin film electrocatalyst by tensile strain. Sci. Rep. $\mathbf{9}, 15906$ (2019). 
4. Stoerzinger, K. A. et al. Highly active epitaxial $\mathrm{La}_{(1-x)} \mathrm{Sr}_{x} \mathrm{MnO}_{3}$ surfaces for the oxygen reduction reaction: role of charge transfer. J. Phys. Chem. Lett. 6, 1435-1440 (2015).

5. Scholz, J. et al. Rotating ring-disk electrode study of oxygen evolution at a perovskite surface: correlating activity to manganese concentration. J. Phys. Chem. C 120, 27746-27756 (2016).

6. Petrie, J. R. et al. Enhanced bifunctional oxygen catalysis in strained $\mathrm{LaNiO}_{3}$ perovskites. J. Am. Chem. Soc. 138, 2488-2491 (2016).

7. Bak, J., Bin H., Oh C., Son J. \& Chung S. Y. Effect of lattice strain on the formation of ruddlesdenpopper faults in heteroepitaxial $\mathrm{LaNiO}_{3}$ for oxygen evolution electrocatalysis. J. Phys. Chem. Lett. 11, 7253-7260 (2020).

8. Liu, J. S. et al. Dynamic lattice oxygen participation on perovskite $\mathrm{LaNiO}_{3}$ during oxygen evolution reaction. J. Phys. Chem. C124, 15386-15390 (2020).

9. Chang, S. H. et al. Activity-stability relationship in the surface electrochemistry of the oxygen evolution reaction. Faraday Discuss. 176, 125-133 (2014).

10. Stoerzinger, K. A., Choi, W. S., Jeen, H., Lee, H. N. \& Shao, H. Y. Role of strain and conductivity in oxygen electrocatalysis on $\mathrm{LaCoO}_{3}$ thin films. J. Phys. Chem. Lett. 6, 487-492 (2015).

11. Wang, L. et al. Strain effect on oxygen evolution reaction activity of epitaxial $\mathrm{NdNiO}_{3}$ thin films. $A C S$ Appl. Mater. Interfaces. 11, 12941-12947 (2019).

12. Choi, M. J. et al. Enhanced oxygen evolution electrocatalysis in strained A site cation deficient $\mathrm{LaNiO}_{3}$ perovskite thin films. Nano Lett. 20, 8040-8045 (2020)

13. Saha, S. \& Ganguli, A. K. FeCoNi alloy as noble metal-free electrocatalyst for oxygen evolution reaction (OER). ChemistrySelect 2, 1630-1636 (2017).

14. Sun, H. M. et al. Self-supported transition-metal-based electrocatalysts for hydrogen and oxygen evolution. Adv. Mater. 32, 1806326 (2019).

15. Ramalingam, V. et al. Heteroatom-mediated interactions between ruthenium single atoms and an MXene support for efficient hydrogen evolution. Adv. Mater. 31, 1903841 (2019).

16. Xia, H., Huang, Z., Lv, C. \& Zhang, C. A self-supported porous hierarchical core-shell nanostructure of cobalt oxide for efficient oxygen evolution reaction. ACS Catal. 7, 8205-8213 (2017).

17. Liao, Z. et al. Controlled lateral anisotropy in correlated manganite heterostructures by interfaceengineered oxygen octahedral coupling. Nature Mater. 15, 425-431 (2016).

18. Jeong, S. G. et al. Propagation control of octahedral tilt in $\mathrm{SrRuO}_{3}$ via artificial heterostructuring. Adv. Sci. 7, 2001643 (2020).

19. Lu, D. et al. Synthesis of freestanding single-crystal perovskite films and heterostructures by etching of sacrificial water-soluble layers. Nat. Mater. 15, 1255-1260 (2016).

20. Dong, G. et al. Super-elastic ferroelectric single-crystal membrane with continuous electric dipole rotation. Science 366, 475-479 (2019). 
21. Ji, D. et al. Freestanding crystalline oxide perovskites down to the monolayer limit. Nature $570,87-$ 90 (2019).

22. Guo, R. et al. Continuously controllable photoconductance in freestanding $\mathrm{BiFeO}_{3}$ by the macroscopic flexoelectric effect. Nat. Commun. 11, 2571 (2020).

23. Dong, G. et al. Periodic wrinkle-patterned single-crystalline ferroelectric oxide membranes with enhanced piezoelectricity. Adv. Mater. 32, 2004477 (2020).

24. Deneke, C. et al. Rolled-up tubes and cantilevers by releasing $\mathrm{SrRuO}_{3}-\mathrm{Pr}_{0.7} \mathrm{Ca}_{0.3} \mathrm{MnO}_{3}$ nanomembranes. Nanoscale Res. Lett. 6, 621 (2011).

25. Hu, C.Z. et al. Strain-controlled electrical and magnetic properties of $\mathrm{SrRuO}_{3}$ thin films with $\mathrm{Sr}_{3} \mathrm{Al}_{2} \mathrm{O}_{6}$ buffer layers. Appl. Phys. Lett. 118, 072407 (2021).

26. Paskiewicz, D. M., Sichel, T. R., Karapetrova, E., Stan, L. \& Fong, D. D. Single-crystalline $\mathrm{SrRuO}_{3}$ nanomembranes: a platform for flexible oxide electronics. Nano Lett. 16, 534-542, (2016).

27. Grutter, A. J., Wong, F. J., Arenholz, E. \& Suzuki, Y. Evidence of high-spin Ru and universal magnetic anisotropy in $\mathrm{SrRuO}_{3}$ thin films. Phys. Rev. B 85.134429 (2012).

28. Akbashev, A. R. et al. Activation of ultrathin $\mathrm{SrTiO}_{3}$ with subsurface $\mathrm{SrRuO}_{3}$ for the oxygen evolution reaction. Energ. Enviro. Sci. 11, 1762-1769, (2018).

29. Lee, S. A. et al. Enhanced electrocatalytic activity via phase transitions in strongly correlated $\mathrm{SrRuO}_{3}$ thin films. Energ. Enviro. Sci. 10, 924-930, (2017).

30. Chang, S.H. et al. Functional links between stability and reactivity of strontium ruthenate single crystals during oxygen evolution. Nat Commun. 5, 4191, (2014).

31. Hirai, S. et al. $\mathrm{Ca}_{1}-{ }_{x} \mathrm{Sr}_{x} \mathrm{RuO}_{3}$ perovskite at the metal-insulator boundary as a highly active oxygen evolution catalyst. J Mater. Chem. A 7, 15387-15394, (2019).

32. Koster, G. et al. Structure, physical properties, and applications of $\mathrm{SrRuO}_{3}$ thin films. Rev. Mod. Phys. 84, 253-298, (2012).

33. Liu, J. D. et al. Mechanically tunable magnetic properties of flexible $\mathrm{SrRuO}_{3}$ epitaxial thin films on mica substrates. Adv. Electron. Mater. 4, 1700522 (2018).

34. Grutter, A. et al. Enhanced magnetism in epitaxial $\mathrm{SrRuO}_{3}$ thin films. Appl. Phys. Lett. 96, 082509 (2010).

35. Suntivich J., May, K. J., Gasteiger, H. A., Goodenough, J. B. \& Shao-Horn, Y. A perovskite oxide optimized for oxygen evolution catalysis from molecular orbital principles. Science 334, 1383-1385 (2011).

36. Lee, J. G. et al. A new family of perovskite catalysts for oxygen-evolution reaction in alkaline media: $\mathrm{BaNiO}_{3}$ and $\mathrm{BaNi}_{0.83} \mathrm{O}_{2.5}$. J. Am. Chem. Soc. 138, 3541-3547 (2016).

37. Yagi, S. et al. Covalency-reinforced oxygen evolution reaction catalyst. Nat. commun. 6, 1-6 (2015).

38. Jung, C. U., Yamada, H., Kawasaki, M. \& Tokura, Y. Magnetic anisotropy control of $\mathrm{SrRuO}_{3}$ films by tunable epitaxial strain. Appl. Phys. Lett. 84, 2590 (2004). 
39. Bern, F. et al. Structural, magnetic and electrical properties of $\mathrm{SrRuO}_{3}$ films and $\mathrm{SrRuO}_{3} / \mathrm{SrTiO}_{3}$ superlattices. J. Phys.-Condens. Mat. 25, 496003 (2013).

40. Lee, S. A. et al. Enhanced electrocatalytic activity via phase transitions in strongly correlated $\mathrm{SrRuO}_{3}$ thin films. Energy Envorin. Sci. 10, 924-930 (2017).

41. Chang, S. H. et al. Functional links between stability and reactivity of strontium ruthenate single crystals during oxygen evolution. Nat. Commun. 5, 4191 (2014).

42. Choi, K. J. et al. Enhancement of ferroelectricity in strained $\mathrm{BaTiO}_{3}$ thin films. Science 306, 10051009, (2004).

43. Pantel, D. \& Alexe, M., Electroresistance effects in ferroelectric tunnel barriers. Phys. Rev. B 82, 134105 (2010).

44. Kim, D. J. et al. Polarization relaxation induced by a depolarization field in ultrathin ferroelectric capacitors. Phys. Rev. Lett. 95, 237602, (2005).

45. Bushmeleva, S. N., Pomjakushin, V. Y., Pomjakushina, E. V., Sheptyakov, D. V. \& Balagurov, A. M. Evidence for the band ferromagnetism in $\mathrm{SrRuO}_{3}$ from neutron diffraction. J Magn. Magn. Mater. 305, 491-496, (2006).

46. Allen, P. B. et al. Transport properties, thermodynamic properties, and electronic structure of $\mathrm{SrRuO}_{3}$. Phys. Rev. B Condens. Matter 53, 4393-4398, (1996).

47. Lu J. et al. Electric field controllable high-spin $\mathrm{SrRuO}_{3}$ driven by a solid ionic junction. Phys. Rev. $B$ 101,214401 (2020).

48. Chen, G. et al. Two orders of magnitude enhancement in oxygen evolution reactivity on amorphous $\mathrm{Ba}_{0.5} \mathrm{Sr}_{0.5} \mathrm{Co}_{0.8} \mathrm{Fe}_{0.2} \mathrm{O}_{3-\delta}$ nanofilms with tunable oxidation state. Sci. Adv. 3, e1603206 (2017).

49. Tachikawa, T., Beniya, A., Shigetoh, K. \& Higashi, S. Relationship between OER activity and annealing temperature of sputterdeposited flat $\mathrm{IrO}_{2}$ thin films. Catal. Lett. 150, 1976-1984 (2020).

\section{Methods}

Sample Preparation: For the (SRO/BTO/SRO) ${ }_{n} / \mathrm{SAO} / \mathrm{SRO} / \mathrm{STO}$ heterostructures ( $n$ is the number of periods), the first SRO $(16 \mathrm{~nm})$ was deposited on a $\mathrm{TiO}_{2}$-terminated STO (001) substrate by the PLD method (KrF laser $\lambda=248 \mathrm{~nm}$ ). Then, SAO (50 nm) sacrificial layer and SRO $(2 \mathrm{~nm}) / \mathrm{BTO}(80 \mathrm{~nm}) / \mathrm{SRO}$ $(16 \mathrm{~nm})$ sandwich heterostructures were deposited on SRO/STO. By repeating the SAO/SRO/BTO/SRO growth, multiple C-SROs with different $n$ can be obtained after the water dissolution. During the PLD deposition, the energy density of the laser beam was adjusted to be $2.3 \mathrm{~J} / \mathrm{cm}^{2}$ for SAO and SRO layers, and $3.0 \mathrm{~J} / \mathrm{cm}^{2}$ for BTO. SRO and BTO layers were deposited at $750{ }^{\circ} \mathrm{C}$ and the oxygen partial pressure was $1 \times 10^{-1}$ Torr, besides the SAO sacrificial layer was deposited under $5 \times 10^{-5}$ Torr at $700{ }^{\circ} \mathrm{C}$. 
Characterizations: The crystal structure of thin films was characterized by X-ray diffraction (XRD) of Rigaku Smartlab (3KW). The optical microscope (DM2700M, Leica) and field emission scanning electron microscope (FESEM, Regulus 8100, HITACHI) were employed to get the optical images and local morphologies of our samples. The X-ray photoelectron spectroscopy measurements (ESCALAB 250, Thermo Fisher Scientific) were carried out using a non-monochromatized Al $K_{\mathrm{a}} \mathrm{X}$-ray source, with a photon energy of $1486.6 \mathrm{eV}$. The out-of-plane magnetic hysteresis loops and magnetic moment vs temperature curves of samples were measured by Superconductor Quantum Interference Device (SQUID). The resistivity as a function of temperature for the SRO films was examined by Physical Property Measurement System (PPMS) of Quantum Design using the standard four-probe method in a temperature range of $10-300 \mathrm{~K}$.

OER measurements: The OER activities of the catalysts were evaluated by a standard three-electrode system in $1 \mathrm{~mol} / \mathrm{L} \mathrm{KOH}$ solution using a Solartron Analytical 1470E, the KOH solutions were purged $\mathrm{N}_{2}$ gas at room temperature before OER measurements. The counter and reference electrodes are respective carbon rod and $\mathrm{Hg} / \mathrm{HgO}$ electrode, and the working electrode was set by fixing our sample on a copper sheet with silver paint. After achieving an Ohmic contact, the working electrode was covered with a chemical resistant epoxy, except an exposed surface. All potentials were converted to a reversible hydrogen electrode $(\mathrm{RHE})$ : $\mathrm{E}$ vs $\mathrm{RHE}=\mathrm{E}$ vs $\mathrm{Hg} / \mathrm{HgO}+0.098+0.059 \times \mathrm{pH}$. All potentials mentioned in this study are versus RHE. LSV measurements were measured at a scan rate of $1 \mathrm{mV} / \mathrm{s}$. The electrochemical $C_{\mathrm{dl}}$ were measured by cyclic voltammetry in a range of 0.924 to $1.024 \mathrm{~V}$ with $80,40,20$, and $10 \mathrm{mV} / \mathrm{s}$. the EIS Nyquist curves were measured at a frequency from $10^{5}$ to $0.01 \mathrm{~Hz}$ at $1.9 \mathrm{~V}$ by the $5 \mathrm{mV}$ amplitude AC signal.

\section{Figures}


a

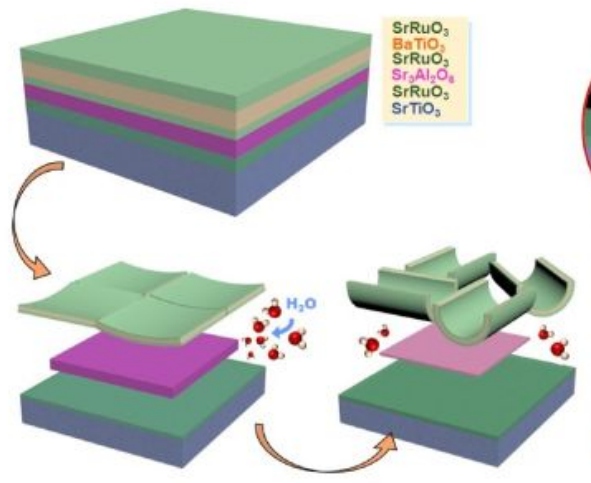

C

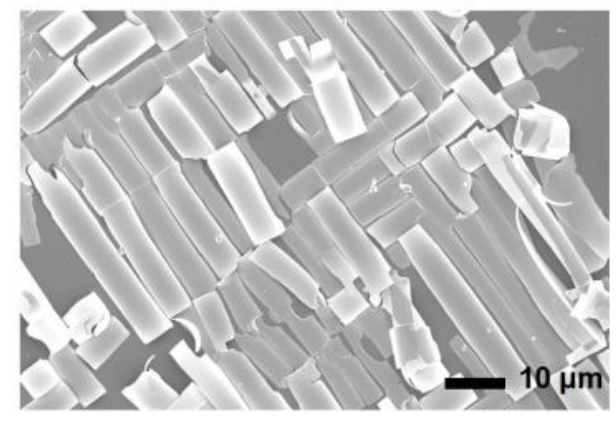

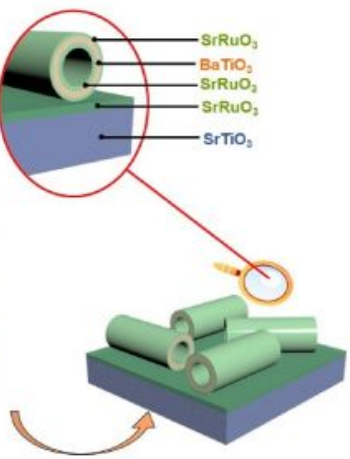

d

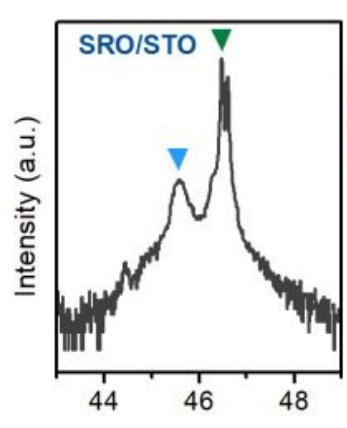

b

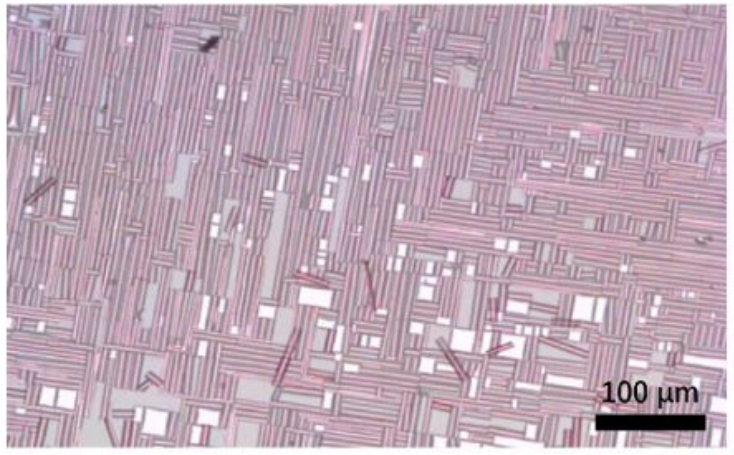

e

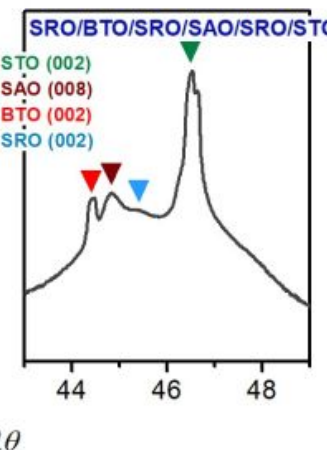

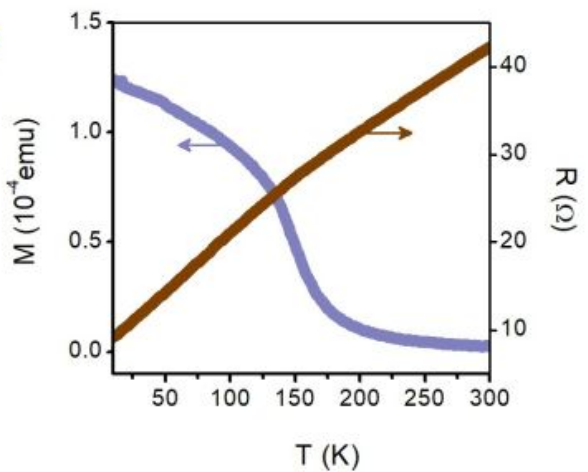

Figure 1

a, The schematic illustration of the achievement of cylindrical shape (SRO/BTO/SRO) $n$ with $n=1$ ( $n$ is the number of periods). $b$, The optical image of the freestanding SRO at the beginning stage of the waterdissolution process. c, The SEM image of the freestanding SRO at the beginning stage of the waterdissolution process. The SROs show outside-in coiling. d, The XRD patterns of rigid SRO/STO and SRO/BTO/SRO/SAO/SRO/STO heterostructures. e, The magnetization under $100 \mathrm{Oe}$, and resistance as a function of the temperature of rigid SRO. 

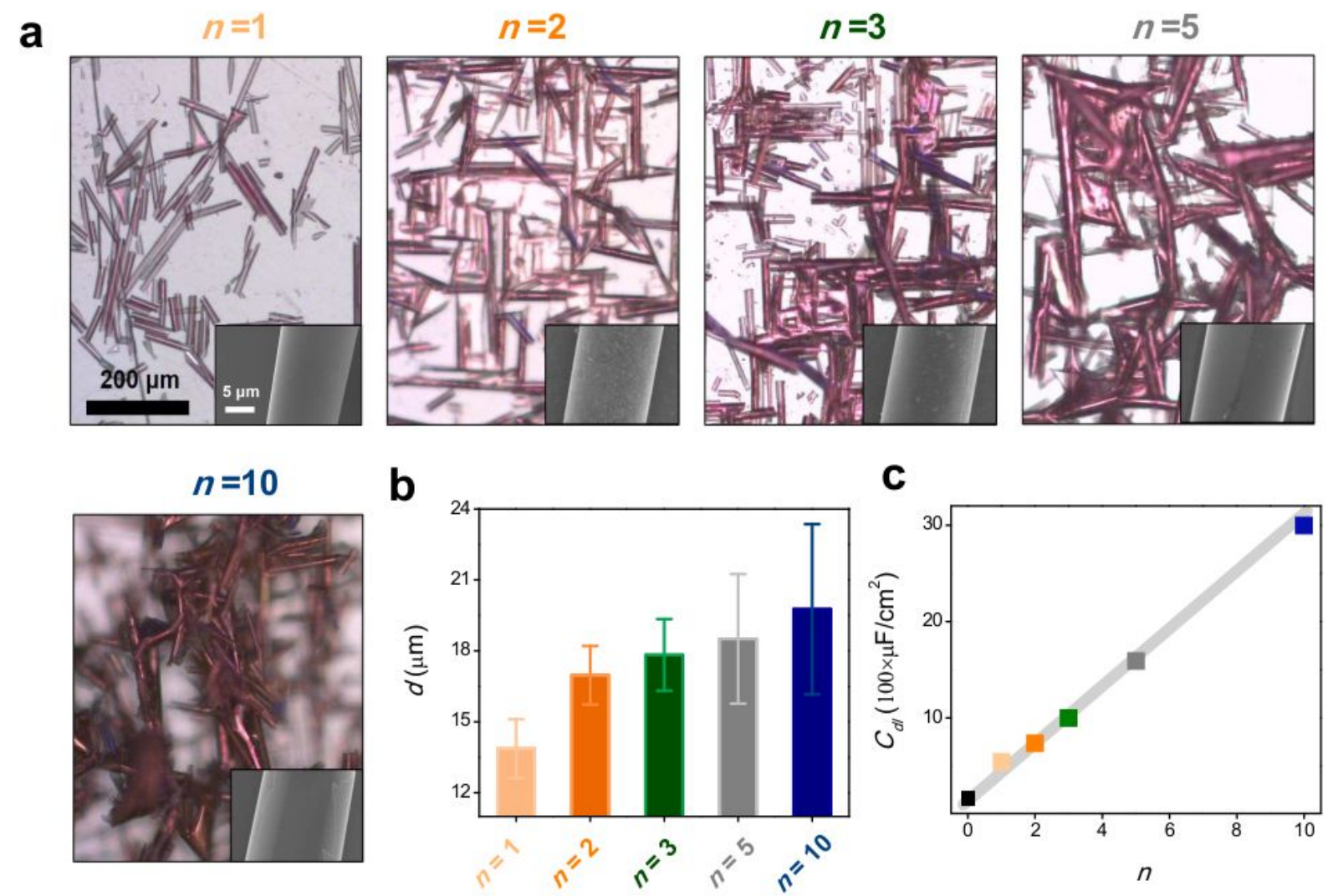

Figure 2

a, The optical and SEM images of C-SRO with different $n$. b, The statistic diameter of C-SRO from $n=1$ to 10. $c$, Cdl as a function of $n$. 
a

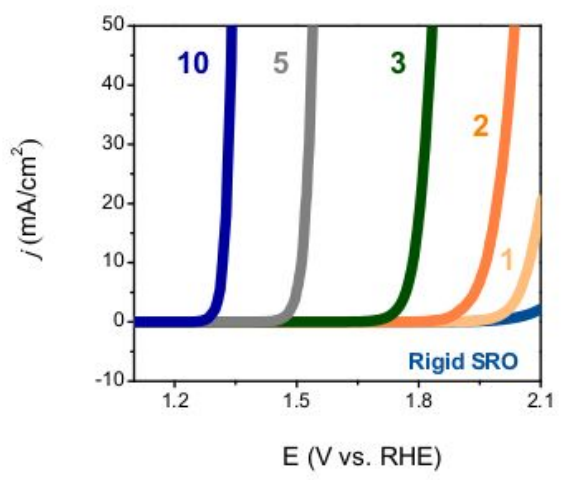

d

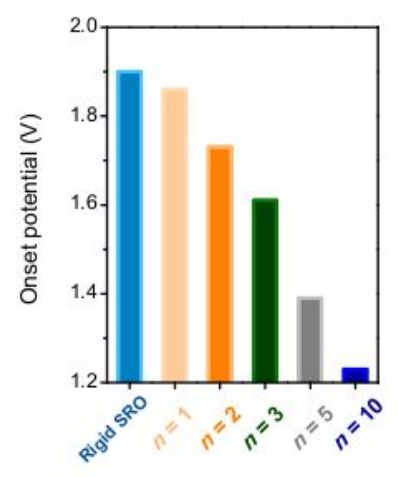

e b
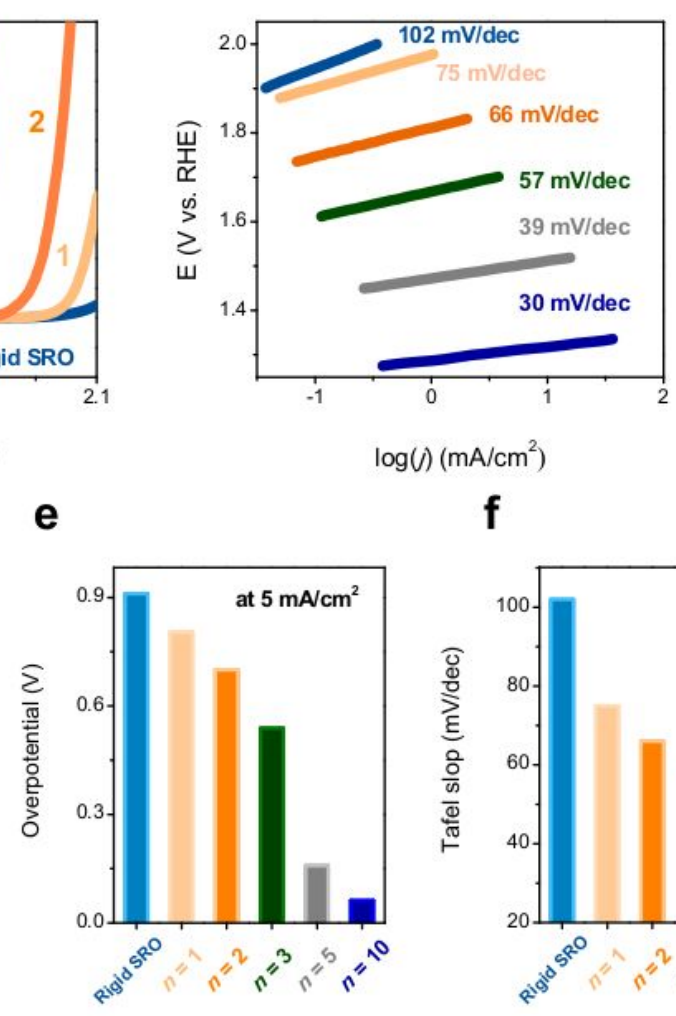

C

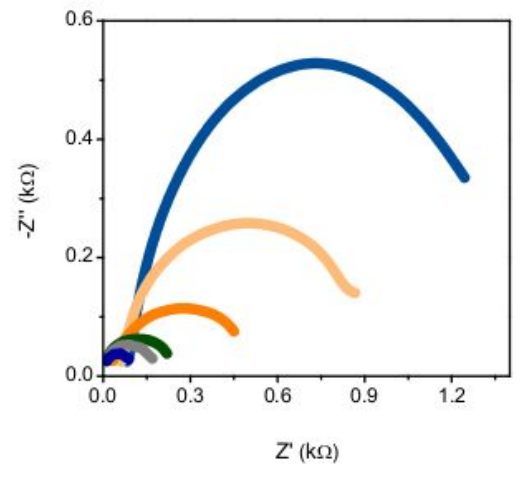

g

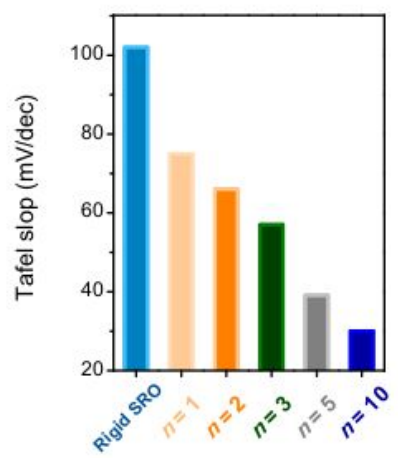

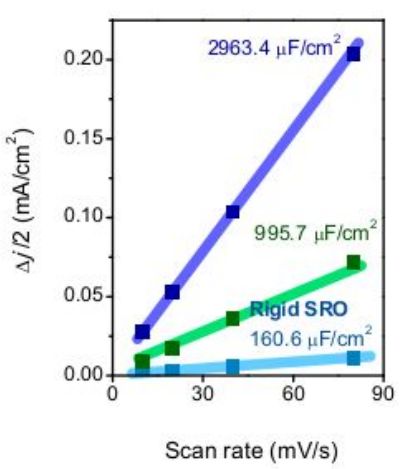

Figure 3

a, The LSV versus electric field, b, Tafel plot, and c, EIS of rigid and C-SRO $(n=1,2,3,5$, and 10$)$ covered SROs. d, e, and f, The summarised onset potential, overpotential, and Tafel slope. g, Linear fitting of the capacitive current densities versus CV scan rates of rigid and C-SRO $(n=0,3$, and 10) covered SROs. 


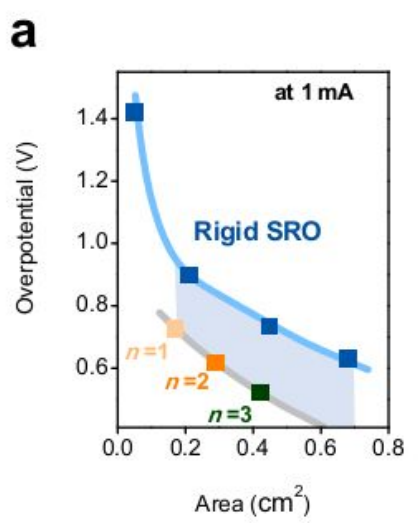

b

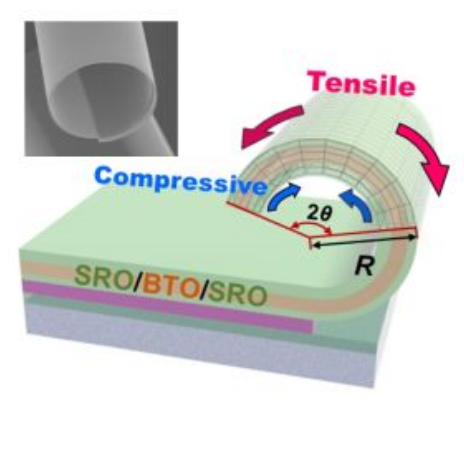

C

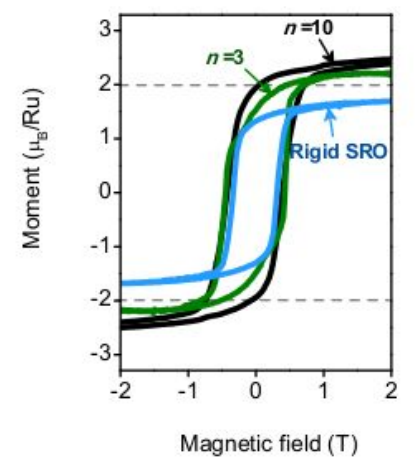

d

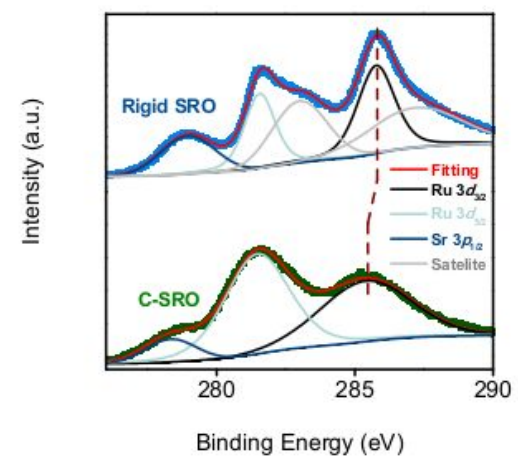

\section{Figure 4}

a, The overpotential at $1 \mathrm{~mA}$ as a function of rigid SRO and C-SRO surface area. The C-SRO area was evaluated by the ideal area from rigid heterostructure before water-dissolution. b, A diagram of the strain condition of inner and outer SROs in C-SRO. c, The moment of Ru versus magnetic field of rigid SRO and C-SRO ( $n=3$ and 10$)$ at $10 \mathrm{~K}$. d, The XPS patterns of rigid SRO and C-SRO respectively.

\section{Supplementary Files}

This is a list of supplementary files associated with this preprint. Click to download.

- SupplementaryInformation.docx 